\title{
Efficacy of Antibiotic Laden Collagen Sponge In Treating Open Fracture
}

Kularaj Subramaniam ${ }^{1,}$ Aminuddin Che Ahmad ${ }^{1}$

${ }^{1}$ Department of Orthopaedics, Traumatology and Rehabilitation, Kulliyyah of Medicine, International Islamic University Malaysia

Presenter: Kularaj Subramaniam

Introduction: Open fractures are known as a major predisposing factor for a higher incidence of infection. The aim of this study was to assess the efficacy of antibiotic impregnated collagen sponge in reducing the risk of infection in open fractures Grade 3. Materials and Methods: This was an observational cohort study, evaluating the incidence of infection in open fractures of the femur Grade 3A/3B following insertion of gentamicin impregnated collagen sponge (Collatamp $\AA$ ) during definitive fracture fixation. Eryhtrocyte Sedimentation Rate (ESR) and total white count (TWC) were used as blood parameters to observe for the possibility of infection from pre-operatively up to follow up visits at the clinic. Results: A total number of 36 patients, whom had sustained open fracture of the femur Grade $3 A / B$, underwent internal fixation with antibiotic impregnated collagen sponge insertion. Overall, ESR and TWC in both male and female gender were downgoing trend, with $p$ values of $<0.001$, and clinically there was no evidence of infection. No infection was identified in $97.2 \%$ of patients, following the internal fixation and antibiotic impregnated collagen sponge insertion. There was only one patient (2.8\%) who developed infection following the definitive internal fixation and antibiotics impregnated collagen sponge insertion. Conclusion: The use of antibiotic impregnated collagen sponge in open fractures reduced the occurrence of infection. Furthermore, this allows for an uncomplicated union of the bone following definitive fixation. 